\title{
Anti-Aminoacyl tRNA Synthetases Antibodies in Japanese Patients with Interstitial Lung Disease
}

\author{
Shinji Sato1, Michito Hirakata ${ }^{2}$, Koichiro Asano³ ${ }^{3}$, Masataka Kuwana ${ }^{4}$ \\ ${ }^{1}$ Division of Rheumatology, Department of Internal Medicine, Tokai University, School of Medicine, Isehara, Japan \\ ${ }^{2}$ Medical Education Center, Keio University School of Medicine, Tokyo, Japan \\ ${ }^{3}$ Division of Pulmonary Medicine, Department of Internal Medicine, Tokai University, School of Medicine, Isehara, Japan \\ ${ }^{4}$ Department of Allergy and Rheumatology, Nippon Medical School Graduate School of Medicine, Tokyo, Japan \\ Email: shinsjam@tokai-u.jp
}

How to cite this paper: Sato, S., Hirakata, M., Asano, K. and Kuwana, M. (2018) Anti-Aminoacyl tRNA Synthetases Antibodies in Japanese Patients with Interstitial Lung Disease. Open Journal of Rheumatology and Autoimmune Diseases, 8, 34-42. https://doi.org/10.4236/ojra.2018.81003

Received: January 3, 2018

Accepted: February 24, 2018

Published: February 27, 2018

Copyright $\odot 2018$ by authors and Scientific Research Publishing Inc. This work is licensed under the Creative Commons Attribution International License (CC BY 4.0).

http://creativecommons.org/licenses/by/4.0/ Open Access

\begin{abstract}
Objectives: In the present study, we have sought to establish the clinical and immunological characteristics of Japanese patients with interstitial lung disease (ILD). Methods: Serum samples from 35 patients of ILD were screened for autoantibodies using RNA and protein immunoprecipitation assays. Patients with or without serum antibodies to aminoacyl tRNA synthetases (ARS) were assessed clinically and compared. Results: Sera from 12 of 35 (34\%) patients with ILD (mean age at onset $=49.7 \mathrm{yrs}$; range $27-65 \mathrm{yrs}$ ) were found to contain anti-ARS antibodies (anti-EJ: 3 patients; anti-OJ: 2 patients; anti-PL-12: 3 patients; anti-KS: 4 patients). Nine of the 12 (75\%) were female. Six (50\%) had Raynaud's phenomenon, 5 (42\%) had arthralgia/arthritis and four (33\%) had rheumatoid factor. Lung biopsy specimens of 8 patients with anti-ARS antibodies were examined histologically in detail. The following was determined: Two patients had usual interstitial pneumonia; 3 had non-specific interstitial pneumonia; one had organizing pneumonia; one had lymphocyte interstitial pneumonia and the remaining patient had desquamative interstitial pneumonia. Age at disease onset was significantly lower and the frequency of Raynaud's phenomenon was significantly greater in these patients compared to anti-ARS-negative patients ( 49.7 yrs vs. $62.6 \mathrm{yrs}, \mathrm{p}=0.004 ; 50 \%$ vs. $4 \%, \mathrm{p}=0.003$, respectively). Conclusions: These results indicate that the presence of anti-ARS autoantibodies correlates with ILD without definite diagnosis of connective tissue diseases as well as polymyositis/dermatomyositis (PM/DM) with ILD in Japanese patients.
\end{abstract}

\section{Keywords}

Interstitial Lung Disease (ILD), Anti-Aminoacyl tRNA Synthetases (ARS)

Antibodies, Autoantibody, Interstitial Pneumonia with Autoimmune 


\section{Introduction}

In patients with idiopathic inflammatory myopathies, several autoantibodies to the 8 aminoacyl-tRNA synthetases (anti-ARS antibodies) histidyl (anti-Jo-1), threonyl (anti-PL-7), alanyl (anti-PL-12), glycyl (anti-EJ), isoleucyl (anti-OJ), asparaginyl (anti-KS), tyrosyl (anti-Ha) and phenylalanyl (anti-Zo) tRNA synthetases have been identified [1]-[11]. Among these, the most common is anti-Jo-1, found in approximately $20 \%-30 \%$ of patients with polymyositis/ dermatomyositis (PM/DM). Patients with different anti-ARS antibodies manifest certain common clinical symptoms (fever, Raynaud's phenomenon, arthritis and chronic interstitial lung disease (ILD)) together designated anti-synthetase syndrome [2]. However, further observation and analysis suggests that each of the anti-ARS antibodies is to some degree associated with different clinical manifestations [2] [8] [12] [13].

It has been known for some time that anti-Jo-1 antibodies are more common in patients with typical PM/DM, whereas anti-PL-12 and anti-KS antibodies are found in patients with ILD without any signs of myositis. Thus, the latter are more likely to be associated with the condition of ILD per se without clinical evidence of myositis. Recently, anti-ARS antibodies have also been reported in patients with idiopathic interstitial pneumonias (IIPs) [13]-[18].

In the present study, we have screened sera from patients with ILD on extracts of HeLa cells in order to identify anti-ARS and other autoantibodies associated with this condition.

\section{Methods}

\subsection{Patients and Sera}

Serum samples from 35 Japanese patients with ILD who did not satisfy the classification criteria of any connective tissue diseases (CTDs) including idiopathic inflammatory myopathies were screened for autoantibodies using immunoprecipitation (IP) assays from 2000 through 2006. The presence of ILD was evaluated by a clinical history, physical examination, chest radiograph/ computed tomography and lung function test based on American Thoracic Society/European Respiratory Society International Multidisciplinary Consensus Classification of the Idiopathic Interstitial Pneumonias [19]. Blood samples were obtained after the patients had provided written informed consent approved by the Keio University Institutional Review Board.

\subsection{Immunoprecipitation (IP) [7]}

The IP assay using HeLa cell extracts was performed for RNAs and protein components separately. Analysis of RNAs: Complexes of antibodies and protein 
A-Sepharose CL-4B beads were incubated with HeLa cells extracts. After washing with NET-2 buffer (50 mM Tris-HCL, pH 7.5, $150 \mathrm{mM} \mathrm{NaCl}, 0.05 \%$ Nonidet $\mathrm{P}-40)$ and ethanol precipitation, RNAs were soluble in sample buffer (10 M urea, $0.025 \%$ bromophenol blue, and $0.025 \%$ xylene cyanol-FF in TBE buffer $(90 \mathrm{mM}$ Tris- $\mathrm{HCl}, \mathrm{pH} 8.6,90 \mathrm{mM}$ boric acid, and $1 \mathrm{mM}$ EDTA)). Then, the RNA samples were fractionated by $7 \mathrm{M}$ urea-10\% polyacrylamide gels and subsequently silver stained (Bio-Rad Laboratories, Hercules, CA).

Analysis of proteins: Sepharose beads were incuvated with patients' sera and then $\left[{ }^{35} \mathrm{~S}\right]$ methionine-labeled HeLa cell extracts were added and washed with IP buffer. Next, the Sepharose beads were added in SDS-sample buffer (2\% SDS, $10 \%$ glycerol, $62.5 \mathrm{mM}$ Tris- $\mathrm{HCl}, \mathrm{pH} 6.8,0.005 \%$ bromophenol blue) and electrophoretically separated by $10 \%$ SDS-PAGE. Finally radiolabeled protein were visualized by autoradiography.

Identification of anti-ARS antibodies was determined by comparing the pattern of IP with anti-ARS prototype sera [3].

\subsection{Clinical Features}

The patients whose sera recognized ARS autoantigen were retrospectively investigated regarding their clinical symptoms, laboratory findings, Uptake in Gallium (Ga) scintigram, lung function testing (percent predicted forced vital capacity [FVC] and carbon monoxide diffusing capacity [DLco]), bronchoalveolar lavage fluid (BALF) findings and pathological findings in the lung by transbronchial lung biopsy (TBLB) or video-assisted thoracoscopic surgery (VATS).

\subsection{Statistical Analysis}

All comparisons between each patient group were performed using the Chi-square test, with Fisher's exact test where appropriate.

\section{Results}

\subsection{Detection of Autoantibodies in Patients with ILD (Figure 1)}

We screened 35 sera of ILD patients by RNA and protein IP assay. Sera from 12 $(34 \%)$ patients (mean age at onset $=49.7$ years; range $27-65$ years) were found to have anti-ARS antibodies (anti-EJ: 3 patients; anti-OJ: 2 patients; anti-PL-12: 3 patients; anti-KS: 4 patients).

\subsection{Clinical Characteristics in Patients with ILD Who Had Anti-ARS Antibodies (Table 1)}

Nine of the $12(75 \%)$ patients studied were female. Six (50\%) had Raynaud's phenomenon, 5 (42\%) had arthralgia/arthritis, 4 (33\%) had rheumatoid factor (RF), 3 (25\%) also had fever and sicca syndrome, and finally, 2 (17\%) also had sclerodactyly and anti-nuclear antibody (ANA). The most frequent ARS antibody was the anti-KS antibody (33\%) with no anti-Jo-1 antibody detected. 


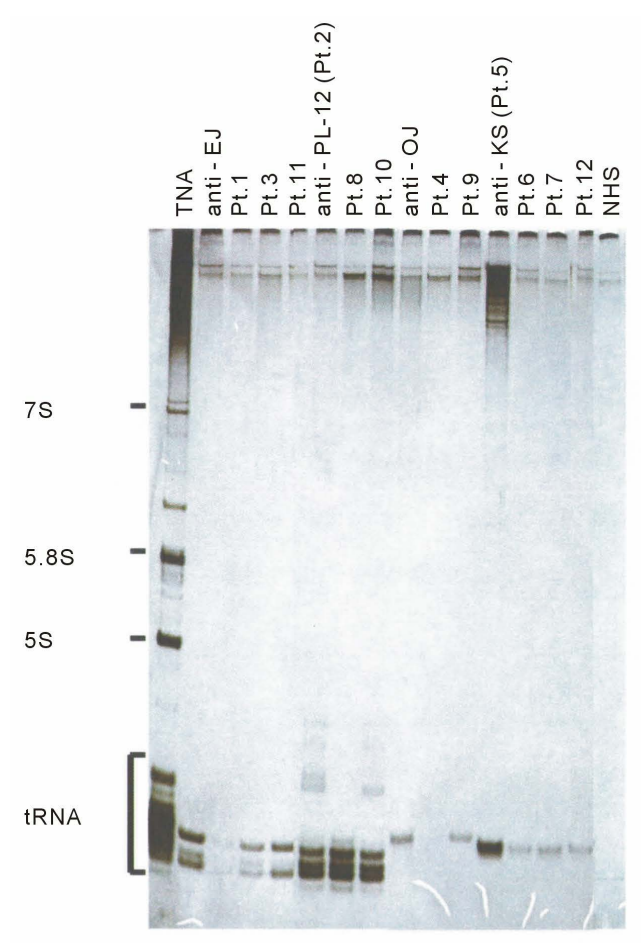

$122 \quad 3 \quad 4 \quad 5 \quad 6 \quad 7 \quad 8 \quad 9101112131415$

(a)

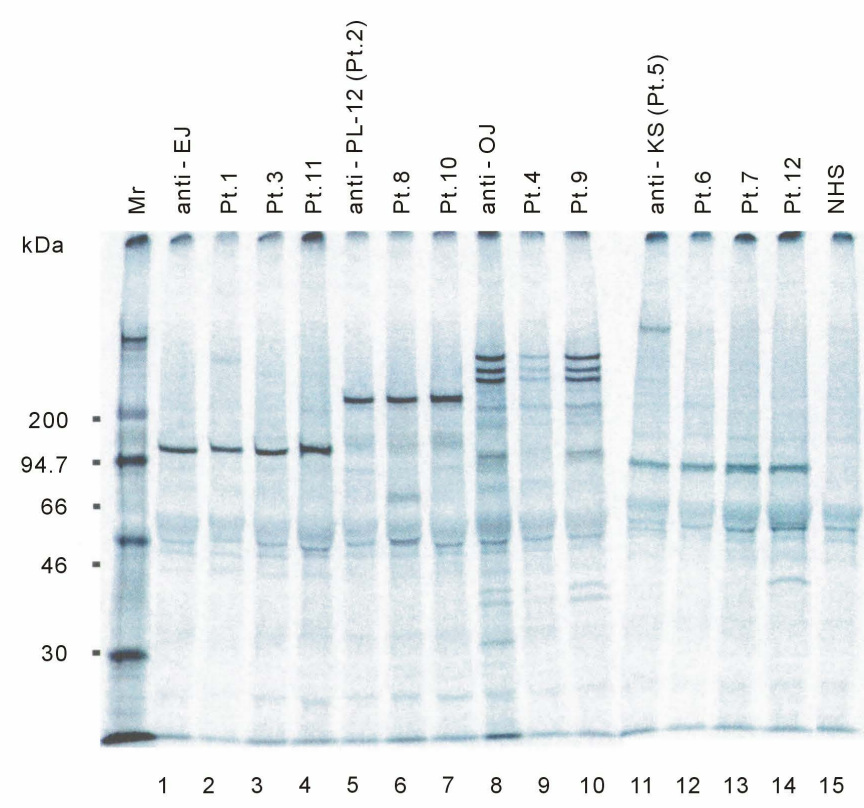

(b)

Figure 1. (a) Immunoprecipitation (IP) of nucleic acids with each anti-ARS sera and controls. Urea (7M) and 10\% PAGE of phenol-extracted immunoprecipitates from HeLa cell extracts were developed with silver stain. TNA, total nucleic acids, with the 7, 5.8 and 5.0 S small ribosomal RNAs and the tRNA region indicated. Sera used for IP include: lanes 1-4, anti-EJ (glycyl-tRNA synthetase) sera indicated; lanes 5-7, anti-PL-12 (alanyl-tRNA synthetase) sera indicated; lanes 8-10, anti-OJ (isoleucyl-tRNA synthetase) sera indicated; lanes 11-14, anti-KS (asparaginyl-tRNA synthetase) sera indicated; and lane 15, control serum indicated (NHS, normal human serum). The tRNA pattern with anti-EJ, PL-12, OJ and KS sera is easily distinguishable from that of the other anti-ARS antibodies. (b) IP of proteins with anti-ARS sera and controls. Autoradiogram of $10 \%$. SDS-PAGE of immunoprecipitates from [ $\left.{ }^{35} \mathrm{~S}\right]$ methionine-labelled HeLa cell extracts. Mr, molecular weight markers of the sizes indicated to the left ( $\mathrm{kDa})$. The sera used for immunoprecipitation are the same as those in Figure 1(a). The same characteristic pattern of protein band was seen with each of the four anti-ARS sera.

\subsection{Pulmonary Characteristics of ILD Patients with Anti-ARS Antibodies (Table 1)}

All patients manifested a restrictive pattern in pulmonary function testing. Serum KL-6 levels (cut off level was $500 \mathrm{U} / \mathrm{mL}$ ) were high in 10 patients (but one patient had no available data). BALF analysis revealed that 4 patients had an elevated lymphocyte ratio ( $>20 \%)$ and 6 had an inverted $\mathrm{CD}^{+} / \mathrm{CD}^{+} \mathrm{T}$ cell ratio (data not shown). Lung biopsy specimens of 8 patients with anti-ARS autoantibodies were examined in detail by histology. It was determined that 3 had non-specific interstitial pneumonia (NSIP), 2 patients had usual interstitial pneumonia (UIP), one had organizing pneumonia (OP), one had Lymphocyte interstitial pneumonia (LIP) and the remaining patient had desquamative interstitial pneumonia (DIP). Six patients were treated with prednisolone alone and one with prednisolone and other immunosuppressants for respiratory symptoms, which were thereby improved in 4 of them (57\%). 
Table 1. Clinical and pulmonary characteristics features of ILD patients with anti-ARS antibodies.

\begin{tabular}{|c|c|c|c|c|c|c|c|c|c|c|c|c|}
\hline No. & 1 & 2 & 3 & 4 & 5 & 6 & 7 & 8 & 9 & 10 & 11 & 12 \\
\hline age/gender & $53 / \mathrm{F}$ & $61 / \mathrm{F}$ & $58 / \mathrm{M}$ & $27 / M$ & $65 / \mathrm{M}$ & $58 / \mathrm{F}$ & $36 / \mathrm{F}$ & $47 / \mathrm{F}$ & $32 / \mathrm{F}$ & $35 / \mathrm{F}$ & $63 / \mathrm{F}$ & $61 / \mathrm{F}$ \\
\hline Fever & $(-)$ & $(+)$ & $(-)$ & $(-)$ & $(-)$ & $(-)$ & $(-)$ & $(+)$ & $(+)$ & $(-)$ & $(-)$ & $(-)$ \\
\hline Skin manifestation & $(-)$ & $(-)$ & $(-)$ & $(-)$ & $(-)$ & $(-)$ & $(-)$ & $(-)$ & $(-)$ & $(-)$ & $(-)$ & $(-)$ \\
\hline Arthritis/arthralgia & $(+)$ & $(-)$ & $(+)$ & $(-)$ & $(+)$ & $(-)$ & $(+)$ & $(-)$ & $(-)$ & $(-)$ & $(+)$ & $(-)$ \\
\hline Muscle weakness & $(-)$ & $(-)$ & $(-)$ & $(-)$ & $(-)$ & $(-)$ & $(+)$ & $(-)$ & $(-)$ & $(-)$ & $(-)$ & $(-)$ \\
\hline Raynaud's phenomenon & $(+)$ & $(+)$ & $(+)$ & $(-)$ & $(-)$ & $(+)$ & $(-)$ & $(+)$ & $(-)$ & $(-)$ & $(-)$ & $(+)$ \\
\hline Sclerodactyly & $(-)$ & $(-)$ & $(-)$ & $(-)$ & $(-)$ & $(+)$ & $(-)$ & $(+)$ & $(-)$ & $(-)$ & $(-)$ & $(-)$ \\
\hline Sicca syndrome & $(-)$ & $(+)$ & $(-)$ & $(-)$ & $(-)$ & $(+)$ & $(-)$ & $(-)$ & $(-)$ & $(-)$ & $(+)$ & $(-)$ \\
\hline ANA & $(+)$ & $(-)$ & $(-)$ & $(-)$ & $(+)$ & $(-)$ & $(-)$ & $(-)$ & $(-)$ & $(-)$ & $(-)$ & $(-)$ \\
\hline $\mathrm{RF}$ & $(-)$ & $(+)$ & $(+)$ & $(-)$ & $(-)$ & $(-)$ & $(+)$ & $(-)$ & $(-)$ & $(-)$ & $(+)$ & $(-)$ \\
\hline Anti-ARS & EJ & PL-12 & EJ & OJ & KS & KS & KS & PL-12 & OJ & PL-12 & EJ & KS \\
\hline Other antibodies & $(-)$ & SSA & $(-)$ & $(-)$ & $(-)$ & $(-)$ & $(-)$ & $(-)$ & $(-)$ & SSA & $(-)$ & $(-)$ \\
\hline$\% \mathrm{VC}(\%)$ & 78 & 65 & 78 & 74 & 67 & 85 & 35 & 56 & 36 & 74 & 86 & n.d. \\
\hline $\begin{array}{c}\text { DLco/VA } \\
\mathrm{ml} / \mathrm{min} / \text { Torr/l) }\end{array}$ & 5.2 & 5.0 & 3.0 & 5.9 & 4.3 & 4.2 & n.a. & 4.5 & 4.1 & 4.2 & 4.9 & n.a. \\
\hline KL-6 (U/ml) & 231 & 1620 & 957 & 1410 & 1470 & 1520 & n.a. & 742 & 1700 & 7080 & 2040 & 1230 \\
\hline Pathological findings & $\mathrm{OP}$ & NSIP & DIP & UIP & n.a. & LIP & n.d. & UIP & NSIP & n.a. & NSIP & n.d. \\
\hline $\begin{array}{l}\text { Uptake in Gallium } \\
\text { scintigram }\end{array}$ & $(+)$ & $(+)$ & $(+)$ & $(+)$ & $(+)$ & $(-)$ & $(+)$ & $(-)$ & n.d. & n.d. & n.d. & n.d. \\
\hline PSL (mg/day) & $(-)$ & 40 & 60 & 60 & 40 & $(-)$ & $(-)$ & $(-)$ & 50 & Pulse/70 & 60 & $(-)$ \\
\hline Immunosuppressant & $(-)$ & $(-)$ & $(-)$ & $(-)$ & $(-)$ & $(-)$ & $(-)$ & $(-)$ & $(-)$ & $(+)$ & $(-)$ & $(-)$ \\
\hline Outcome & $\mathrm{nc}^{\mathrm{a}}$ & $\mathrm{slp}^{\mathrm{b}}$ & $\mathrm{nc}$ & $i m p^{c}$ & slp & $\mathrm{nc}$ & $\operatorname{slp}$ & $\mathrm{nc}$ & imp & imp & imp & $\mathrm{nc}$ \\
\hline
\end{tabular}

ANA: anti-nuclear antibodies, RF: rheumatoid factor, UIP: Usual interstitial pneumonia, NSIP: non-specific interstitial pneumonia, OP: organizing pneumonia, DIP: desquamative interstitial pneumonia, n.a.: not available, n.d.: not done, Pulse: steroid pulse therapy, ${ }^{a}$ nc: no change, ${ }^{b}$ slp: slowly progressive, cimp: improved.

\subsection{Comparison of Clinical Features in ILD Patients with or without Anti-ARS Antibodies (Table 2)}

Age at disease onset was significantly lower and the frequency of Raynaud's phenomenon was significantly higher in patients with anti-ARS antibodies than in those without ( 49.7 yrs vs. 62.6 yrs: $\mathrm{p}=0.004,50 \%$ vs. $4 \%: \mathrm{p}=0.003$, respectively). Patients who were judged as UIP histopathologically were lower in anti-ARS positive group although there was no statistically significance $(25 \%$ vs. 59\%: $\mathrm{P}=0.20$ )

\section{Discussion}

We have determined that $34 \%$ of Japanese patients with ILD had anti-ARS antibodies. This is relatively high compared with previous reports for IIPs [13] [15], highlighting the importance of anti-ARS testing in patients with ILD as well as $\mathrm{PM} / \mathrm{DM}$ with ILD. 
Table 2. Comparison of clinical features of ILD patients with or without anti-ARS antibodies.

\begin{tabular}{|c|c|c|c|}
\hline & Anti-ARS positive $(\mathrm{n}=12)$ & Anti-ARS negative $(\mathrm{n}=23)$ & $P$ value \\
\hline Age at onset (mean \pm SD) & $49.7 \pm 13.7$ & $62.6 \pm 10.6$ & 0.004 \\
\hline Male/ female & $3 / 9$ & $12 / 11$ & 0.16 \\
\hline Fever $(\%)$ & 25 & 17 & 0.67 \\
\hline Arthralgia/Arthritis (\%) & 42 & 35 & 0.73 \\
\hline Raynaud's phenomenon (\%) & 50 & 4 & 0.003 \\
\hline Sclerodactyly (\%) & 17 & 13 & 1.00 \\
\hline Sicca syndrome(\%) & 25 & 9 & 0.31 \\
\hline KL-6 elevation (\%) & 91 & 87 & 1.00 \\
\hline ANA (\%) & 17 & 17 & 1.00 \\
\hline $\mathrm{RF}(\%)$ & 33 & 22 & 0.69 \\
\hline Histopathologically UIP (\%, n/total available data) & $25(2 / 8)$ & $59(10 / 17)$ & 0.20 \\
\hline
\end{tabular}

ANA: anti-nuclear antibodies, RF: rheumatoid factor, UIP: Usual interstitial pneumonia.

Patients who possess ILD sometimes show clinical features of CTDs and/or immunological laboratory findings but do not satisfy the classification criteria of CTDs [15] [16] [17] [18]. In 2010, Fischer et al. have proposed the terminology of "lung-dominant CTD" for patients with IIPs with CTDs features (i.e. IIPs without definite diagnosis of CTDs) [20]. Recently, European Respiratory Society/American Thoracic Society Research made a statement to use the terminology of "interstitial pneumonia with autoimmune features (IPAF)" for the same IIPs subset [21] [22]. Our series of ILD with anti-ARS antibodies almost fulfill the consensus diagnostic criteria of IPAF (Table 1) although not strictly because some patients were not performed HRCT or surgical lung biopsy.

Several reports have noted strong associations between the presence of anti-ARS antibodies, especially anti-OJ, anti-PL-12 and anti-KS antibodies, and ILD [2] [8] [12]. In our series, anti-KS antibodies were the most frequent with anti-PL-12 and anti-EJ next highest in frequency in patients with ILD. These findings are similar to previous reports investigating anti-ARS antibodies in patients with IIPs, where anti-EJ and anti-PL-12 antibodies were found to be present at high frequency in three reports [13] [15] [16] with 2 cases of anti-KS in another study [14].

Regarding comparisons of clinical features between anti-ARS-positive and -negative ILD patients, a distinguishing feature of the former was a high frequency of Raynaud's phenomenon (but no differences in frequency of other clinical features characteristic of anti-ARS syndrome (fever, arthralgia/arthritis and ILD)). Another distinctive clinical feature was younger onset in anti-ARS-positive patients, also similar to findings in a previous report for IIPs [15]. These observations suggest that anti-ARS antibodies might be associated with ILD patients without definite diagnosis of CTDs occurring at a younger age together with Raynaud's phenomenon and these patients might become definite 
PM/DM during long-term observation. Moreover, It might be possibly exist ILD patients without definite diagnosis of CTDs among patients who was diagnosed as IIPs especially in patients with younger age and good responses to steroids or immunosuppressants. Because the sample size of this study was small, further accumulation of samples would be required to confirm these observations.

It has been reported that pathological findings on lung biopsies from IIPs patients with anti-ARS antibodies are mainly cellular or fibrotic NSIP [15] [16] [17]. However, our pathological diagnosis showed a wide variety of phenomena and there were no distinct trends towards a high frequency of NSIP. Any discrepancy between our findings and previous reports might be due to the small sample size in our study. To clarify characteristic pathological lung findings in patients with anti-ARS-positive ILD requires further study.

\section{Conclusion}

These results indicate that anti-ARS autoantibodies are found in association with ILD without definite diagnosis of CTDs as well as PM/DM with ILD in Japanese patients and may be linked to ILD occurring at younger age together with Raynaud's phenomenon. Further careful observation will be needed to clarify the true characteristics of anti-ARS-positive patients who manifest solely respiratory symptoms.

\section{Acknowledgements}

We thank Ms. Mutsuko Ishida for assisting in RNA immunoprecipitation assays.

\section{Disclosures}

All the co-authors declare that they have no conflict of interest.

\section{References}

[1] Targoff, I.N. (2002) Laboratory Testing in the Diagnosis and Management of Idiopathic Inflammatory Myopathies. Rheumatic Diseases Clinics of North America, 28, 859-890. https://doi.org/10.1016/S0889-857X(02)00032-7

[2] Mimori, T., Nakashima, R. and Hosono, Y. (2012) Interstitial Lung Disease in Myositis: Clinical Subsets, Biomarkers, and Treatment. Current Rheumatology Reports, 14, 264-274. https://doi.org/10.1007/s11926-012-0246-6

[3] Hirakata, M., Mimori, T., Akizuki, M., Craft, J., Hardin, J.A. and Homma, M. (1992) Autoantibodies to Small Nuclear and Cytoplasmic Ribonucleoproteins in Japanese Patients with Inflammatory Muscle Disease. Arthritis \& Rheumatology, 35, 449-456. https://doi.org/10.1002/art.1780350415

[4] Yoshida, S., Akizuki, M., Mimori, T., Yamagata, H., Inada, S. and Homma, M. (1983) The Precipitating Antibody to an Acidic Nuclear Protein Antigen, the Jo-1, in Connective Tissue Diseases. A Maker for a Subset of Polymyositis with Interstitial Pulmonary Fibrosis. Arthritis \& Rheumatology, 26, 604-611. https://doi.org/10.1002/art.1780260505

[5] Mathews, M.B., Reichlin, M., Hughes, G.R. and Bernstein, R.M. (1984) Anti-Threonyl-tRNA Synthetase, a Second Myositis-Related Autoantibody. The Jour- 
nal of Experimental Medicine, 160, 420-434. https://doi.org/10.1084/jem.160.2.420

[6] Bunn, C.C., Bernstein, R.M. and Mathews, M.B. (1986) Autoantibodies against Alanyl-tRNA Synthetase and tRNA ${ }^{\text {Ala }}$ Coexist and Are Associated with Myositis. The Journal of Experimental Medicine, 163, 1281-1291. https://doi.org/10.1084/jem.163.5.1281

[7] Targoff, I.N. (1990) Autoantibodies to Aminoacyl-Transfer RNA Synthetases for Isoleucine and Glycine. Two Additional Synthetases Are Antigenic in Myositis. The Journal of Immunology, 144, 1737-1743.

[8] Hirakata, M., Suwa, A., Nagai, S., Kron, M.A., Trieu, E.P., Mimori, T., Akizuki, M. and Targoff, I.N. (1999) Anti-KS: Identification of Autoantibodies to Asparaginyl-Transfer RNA Synthetase Associated with Interstitial Lung Disease. Journal of Immunology, 162, 2315-2320.

[9] Hervier, B., Devilliers, H., Stanciu, R., Meyer, A., Uzunhan, Y., Masseau, A., Dubucquoi, S., Hatron, P.Y., Musset, L., Wallaert, B., Nunes, H., Maisonobe, T., Olsson, N.O., Adoue, D., Arlet, P., Sibilia, J., Guiguet, M., Lauque, D., Amoura, Z., Hachulla, E., Hamidou, M. and Benveniste, O. (2012) Hierarchical Cluster and Survival Analyses of Antisynthetase Syndrome: Phenotype and Outcome Are Correlated with Anti-tRNA Synthetase Antibody Specificity. Autoimmunity Reviews, 12, 210-217. https://doi.org/10.1016/j.autrev.2012.06.006

[10] Hashish, L., Trieu, E.P., Sadanandan, P. and Targoff, I.N. (2005) Identification of Autoantibodies to Tyrosyl-tRNA Synthetase in Dermatomyositis with Features Consistent with Anti-Synthetase Syndrome. Arthritis \& Rheumatology, 53, S312.

[11] Betteridge, Z., Gunawardena, H., North, J., Slinn, J. and McHugh, N. (2007) Anti-Synthetase Syndrome: A New Autoantibody to Phenylalanyl Transfer RNA Synthetase (Anti-Zo) Associated with Polymyositis and Interstitial Pneumonia. Rheumatology (Oxford), 46, 1005-1008. https://doi.org/10.1093/rheumatology/kem045

[12] Sato, S., Kuwana, M. and Hirakata, M. (2007) Clinical Characteristics of Japanese Patients with Anti-OJ (Anti-Isoleucyl-tRNA Synthetase) Autoantibodies. Rheumatology (Oxford), 46, 842-845. https://doi.org/10.1093/rheumatology/kel435

[13] Matsushita, T., Hasegawa, M., Fujimoto, M., Hamaguchi, Y., Komura, K., Hirano, T., Horikawa, M., Kondo, M., Orito, H., Kaji, K., Saito, Y., Matsushita, Y., Kawara, S., Yasui, M., Seishima, M., Ozaki, S., Kuwana, M., Ogawa, F., Sato, S. and Takehara, K. (2007) Clinical Evaluation of Anti-Aminoacyl tRNA Synthetase Antibodies in Japanese Patients with Dermatomyositis. The Journal of Rheumatology, 34, 1012-1018.

[14] Okayasu, K., Ohtani, Y., Takemura, T., Uchibori, K., Tamaoka, M., Furuiye, M., Miyazaki, Y., Inase, N. and Yoshizawa, Y. (2009) Nonspecific Interstitial Pneumonia (NSIP) Associated with Anti-KS Antibody: Differentiation from Idiopathic NSIP. Internal Medicine, 48, 1301-1306.

https://doi.org/10.2169/internalmedicine.48.2207

[15] Watanabe, K., Handa, T., Tanizawa, K., Hosono, Y., Taguchi, Y., Noma, S., Kobashi, Y., Kubo, T., Aihara, K., Chin, K., Nagai, S., Mimori, T. and Mishima, M. (2011) Detection of Antisynthetase Syndrome in Patients with Idiopathic Interstitial Pneumonias. Respiratory Medicine, 105, 1238-1247.

https://doi.org/10.1016/j.rmed.2011.03.022

[16] Takato, H., Waseda, Y., Watanabe, S., Inuzuka, K., Katayama, N., Ichikawa, Y., Yasui, M. and Fujimura, M. (2013) Pulmonary Manifestations of Anti-ARS Antibody Positive Interstitial Pneumonia-with or without PM/DM. Respiratory Medicine, 107, 128-133. https://doi.org/10.1016/j.rmed.2012.09.005 
[17] Koreeda, Y., Higashimoto, I., Yamamoto, M., Takahashi, M., Kaji, K., Fujimoto, M., Kuwana, M. and Fukuda, Y. (2010) Clinical and Pathological Findings of Interstitial Lung Disease Patients with Anti-Aminoacyl-tRNA Synthetase Autoantibodies. Internal Medicine, 49, 361-369. https://doi.org/10.2169/internalmedicine.49.2889

[18] Hamaguchi, Y., Fujimoto, M., Matsushita, T., Kaji, K., Komura, K., Hasegawa, M., Kodera, M., Muroi, E., Fujikawa, K., Seishima, M., Yamada, H., Yamada, R., Sato, S., Takehara, K. and Kuwana, M. (2013) Common and Distinct Clinical Features in Adult Patients with Anti-Aminoacyl-tRNA Synthetase Antibodies: Heterogeneity within the Syndrome. PLoS ONE, 8, e60442.

https://doi.org/10.1371/journal.pone.0060442

[19] American Thoracic Society; European Respiratory Society (2002) American Thoracic Society/European Respiratory Society International Multidisciplinary Consensus Classification of the Idiopathic Interstitial Pneumonias. American Journal of Respiratory and Critical Care Medicine, 165, 277-304.

[20] Fischer, A., West, S.G., Swigris, J.J., Brown, K.K. and du Bois, R.M. (2010) Connective Tissue Disease-Associated Interstitial Lung Disease: A Call for Clarification. Chest, 138, 251-256. https://doi.org/10.1378/chest.10-0194

[21] Fischer, A., Antoniou, K.M., Brown, K.K., Cadranel, J., Corte, T.J., du Bois, R.M., Lee, J.S., Leslie, K.O., Lynch, D.A., Matteson, E.L., Mosca, M., Noth, I., Richeldi, L., Strek, M.E., Swigris, J.J., Wells, A.U., West, S.G., Collard, H.R., Cottin, V., ERS/ATS Task Force on Undifferentiated Forms of CTD-ILD (2015) An Official European Respiratory Society/American Thoracic Society Research Statement: Interstitial Pneumonia with Autoimmune Features. The European Respiratory Journal, 46, 976-987.

[22] Cottin, V. (2016) Idiopathic Interstitial Pneumonias with Connective Tissue Diseases Features: A Review. Respirology, 21, 245-258.

https://doi.org/10.1111/resp.12588 